\title{
DIMENSIONS OF SPACES OF SIEGEL MODULAR FORMS OF LOW WEIGHT IN DEGREE FOUR
}

\author{
Cris Poor and David S. YUen
}

We calculate the dimensions of $M_{4}^{12}, M_{4}^{8}, S_{4}^{12}, S_{4}^{8}, S_{4}^{6}$ using Erokhin's work on Niemeier lattices and geometric methods involving the hyperelliptic locus.

\section{INTRODUCTION.}

In this note we calculate some dimensions of spaces of Siegel modular forms and of cusp forms. We obtain the results that $\operatorname{dim} M_{4}^{12}=6, \operatorname{dim} M_{4}^{8}=2, \operatorname{dim} S_{4}^{12}=2$, $\operatorname{dim} S_{4}^{8}=1$, and $\operatorname{dim} S_{4}^{6}=0$. Explicit generators for these spaces of cusp forms are also given. The dimensions of the $M_{g}^{k}$ are known for $g \leqslant 3$ [14]; previously it was known [6, p.50] only that $S_{4}^{k}=0$ for $1 \leqslant k \leqslant 5$. Calculations of Erokhin for the Niemeier lattices play an essential role in our arguments, as does a theorem of Igusa that elements of $S_{4}^{k}$ for even $k \leqslant 8$ must vanish on the hyperelliptic locus. The admittedly special nature of these calculations in weights less than or equal to 12 has a natural origin. The Type II lattices in dimension 24 are 24 in number and have been classified by Niemeier, whereas the Type II lattices in dimension 32 number in excess of 80 million and will never be classified in the same detail. A complete classification is essential for applications to Siegel modular forms if Böcherer's result (Theorem 1.2) is to be applied. On the other hand, these dimensions are notoriously difficult to calculate by any means (see [13, pp.60-61]), and this note provides more data toward this famous problem.

The results on cusp forms may be summarised as follows. The theta series for the Niemeier lattices provide a basis for $S_{4}^{12}$ and the study of $S_{4}^{8}$ and $S_{4}^{6}$ is reduced to $S_{4}^{12}$ via the inclusions $M_{4}^{4} S_{4}^{8} \subseteq S_{4}^{12}$ and $S_{4}^{6} S_{4}^{6} \subseteq S_{4}^{12}$. Let $f_{4} \in M_{4}^{4}$ be the theta series of the $E_{8}$ lattice and let $j_{8} \in S_{4}^{8}$ be Schottky's modular form vanishing on the Jacobian

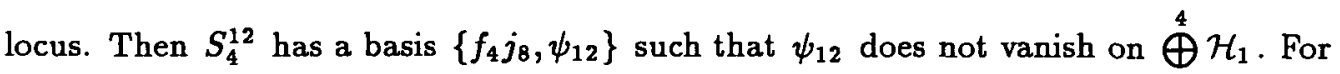
any $e \in S_{4}^{8}$ and $f \in S_{4}^{6}$ we then have $f_{4} e=a f_{4} j_{8}+b \psi_{12}$ and $f^{2}=\alpha f_{4} j_{8}+\beta \psi_{12}$. Since $e$ and $f$ necessarily vanish on the hyperelliptic locus we can evaluate the coefficients $a, b, \alpha, \beta$ by restriction to $\bigoplus^{4} \mathcal{H}_{1}$. We obtain $S_{4}^{8}=\mathbb{C} j_{8}$ and $S_{4}^{6}=0$ by this procedure. This note is another example using geometric information to calculate the dimensions of spaces of cusp forms.

Received 9th November, 1995

Copyright Clearance Centre, Inc. Serial-fee code: 0004-9729/96 \$A2.00+0.00. 


\section{Notation.}

Let $\mathcal{H}_{g}$ be the Siegel upper half space of degree $g \geqslant 1[12, \mathrm{p} .2]$ and let $\Gamma_{g}=\mathrm{Sp}_{g}(\mathbb{Z})$ denote the full Siegel modular group which acts on $\mathcal{H}_{g}$. Let $M_{g}^{k}$ be the complex vector space of Siegel modular forms of weight $k$ on $\mathcal{H}_{g}\left[12\right.$, p.43], $\Phi_{g}: M_{g}^{k} \rightarrow M_{g-1}^{k}$ be the Siegel map [12, p.54], and $S_{g}^{k}=\operatorname{ker} \Phi_{g}$ be the subspace of cusp forms.

To any integral lattice $\Lambda \subseteq \mathbb{R}^{n}$ we may define the theta series of $\Lambda$ (the analytic class invariant) $\vartheta_{\Lambda}: \mathcal{H}_{g} \rightarrow \mathbb{C}$ as follows: for $\Omega \in \mathcal{H}_{g}$ let

$$
\vartheta_{\Lambda}(\Omega)=\sum_{\ell_{1}, \ldots, \ell_{g} \in \Lambda} \exp \left(i \pi \sum_{j, k=1}^{g} \Omega_{j k}\left\langle\ell_{j}, \ell_{k}\right\rangle\right) .
$$

A lattice is called "Type II" $[2$, p.48 $]$ if it is even and self dual. For $\Lambda$ of Type II, we have $\vartheta_{\Lambda} \in M_{g}^{n / 2}$ for each $g \geqslant 1[6, p .17]$. We specify lattices in the notation of [2, p.119, 120, 407] and use the further designations:

$$
\begin{aligned}
& f_{4}=\vartheta_{E_{8}}, \\
& f_{8}=\vartheta_{D_{18}^{+}}, \\
& j_{8}=f_{4}^{2}-f_{8} .
\end{aligned}
$$

Also, for the 24 Niemeier lattices, we denote their theta series by

$$
\vartheta_{i} \in M_{g}^{12} \text { for } i \in\{0,1, \ldots, 23\},
$$

where we index the Niemeier lattices by 0 through 23 in some manner with the Leech lattice being the $0^{\text {th }}$ one; that is, $\vartheta_{0}$ is theta series of the Leech lattice. Let $h_{i}$ be the Coxeter number $\left[2\right.$, p.407] of the $i \underline{t h}$ Niemeier lattice and let $\tau_{i}=24 h_{i}$. Except for the Leech lattice, where $\tau_{0}=0, \tau_{i}$ is the kissing number of the $i^{\underline{t h}}$ Niemeier lattice. The following Theorem is due to Böcherer $[1, \mathrm{p} .22,44]$.

ThEOREM 1.2. (Böcherer) For $k>2 g$ and $k \equiv 0 \bmod 4$, the theta series of all Type II lattices span $M_{g}^{k}$.

This Theorem assures us, for example, that the $\vartheta_{i} \operatorname{span} M_{g}^{12}$ for $1 \leqslant g \leqslant 5$.

We now recall some geometrically defined subsets of $\mathcal{H}_{4} / \Gamma_{4}$ that we shall use. View $\mathcal{A}_{g}=\mathcal{H}_{g} / \Gamma_{g}$ as the moduli space of principally polarised Abelian varieties. The Torelli map sends a compact Riemann surface of genus $g$ to its Jacobian's class in $\mathcal{A}_{g}$. The closure in $\mathcal{A}_{g}$ of the image of the Torelli map is called the Jacobian locus, Jac . Similarly, the closure of the image of the restriction of the Torelli map to hyperelliptic Riemann surfaces is called the hyperelliptic locus, $h_{g}$. We call the image of $\stackrel{g}{\oplus} \mathcal{H}_{1}$ in $\mathcal{A}_{g}$ the diagonal locus, Diag . The following inclusions hold:

$$
\mathcal{A}_{g} \supseteq \mathrm{Jac}_{g} \supseteq h_{g} \supseteq \operatorname{Diag}_{g} .
$$


The final inclusion follows easily from the techniques for the degeneration of curves in [5]. The following theorems of Igusa relate the above loci to the ring of Siegel modular forms. We say that a Siegel modular form $f$ vanishes on $h_{g}$ if for all $\Omega \in \mathcal{H}_{g}$ such that $[\Omega] \in h_{g}$ we have $f(\Omega)=0$.

TheOREM 1.3. (Igusa, $[9, \mathrm{p} .845]) A$ cusp form in $S_{g}^{k}$ of even weight $k<8+4 / g$ necessarily vanishes on the hyperelliptic locus $h_{g}$.

Theorem 1.4. (Igusa, $[\mathbf{1 0}, 11])$ The ideal of Siegel modular forms in $\bigoplus_{k=1}^{\infty} M_{4}^{k}$ that vanish on the Jacobian locus, $\mathrm{Jac}_{4}$, is principal and is generated by the irreducible element $j_{8}$.

\section{Dimension Calculations.}

We begin by constructing certain cusp forms of weight 12 for $1 \leqslant g \leqslant 4$ with simple behaviour on direct sums of $\mathcal{H}_{1}$. We let $\Delta \in S_{1}^{12}$ denote the usual generator of $S_{1}^{12}[2$, p.105]. We also use the following notation:

$$
\underset{i, j, k, l}{\operatorname{det}}\left(\vartheta, \tau^{2}, \tau, 1\right)=\left|\begin{array}{cccc}
\vartheta_{i} & \vartheta_{j} & \vartheta_{k} & \vartheta_{l} \\
\tau_{i}^{2} & \tau_{j}^{2} & \tau_{k}^{2} & \tau_{l}^{2} \\
\tau_{i} & \tau_{j} & \tau_{k} & \tau_{l} \\
1 & 1 & 1 & 1
\end{array}\right| .
$$

Lemma 2.1. For all $i, j, k, l, m \in\{0,1, \ldots, 23\}$ we have

$$
\begin{aligned}
& \operatorname{det}_{i, j}(\vartheta, 1) \in S_{1}^{12} \text { and } \operatorname{det}_{i, j}(\vartheta, 1)=\operatorname{det}_{i, j}(\tau, 1) \Delta \text {, } \\
& \operatorname{det}_{i, j, k}(\vartheta, \tau, 1) \in S_{2}^{12} \text { and }\left.\operatorname{det}_{i, j, k}(\vartheta, \tau, 1)\right|_{\bigoplus^{2} \mathcal{H}_{1}}=\operatorname{det}_{i, j, k}\left(\tau^{2}, \tau, 1\right) \Delta \otimes \Delta \text {, } \\
& \operatorname{det}_{i, j, k, l}\left(\vartheta, \tau^{2}, \tau, 1\right) \in S_{3}^{12} \text { and }\left.\operatorname{det}_{i, j, k, l}\left(\vartheta, \tau^{2}, \tau, 1\right)\right|_{\bigoplus^{3} \mathcal{H}_{1}}=\operatorname{det}_{i, j, k, l}\left(\tau^{3}, \tau^{2}, \tau, 1\right) \Delta \otimes \Delta \otimes \Delta \text {, } \\
& \operatorname{det}_{i, j, k, l, m}\left(\vartheta, \tau^{3}, \tau^{2}, \tau, 1\right) \in S_{4}^{12} \text { and }\left.\operatorname{det}_{i, j, k, l, m}\left(\vartheta, \tau^{3}, \tau^{2}, \tau, 1\right)\right|_{\bigoplus^{4} \mathcal{H}_{1}} \\
& =\operatorname{det}_{i, j, k, l, m}\left(\tau^{4}, \tau^{3}, \tau^{2}, \tau, 1\right) \Delta \otimes \Delta \otimes \Delta \otimes \Delta \text {. }
\end{aligned}
$$

Proof: Recall that for $\Omega \in \mathcal{H}_{1}$, the coefficient of $e^{2 \pi i \Omega}$ in the theta series $\vartheta_{i}(\Omega)$ is $\tau_{i}$, and the coefficient of $e^{2 \pi i n}$ in $\Delta(\Omega)$ is 1 . Since $\vartheta_{i}-\vartheta_{j} \in S_{1}^{12}$ and $\Delta$ is a generator of $S_{1}^{12}$, we must have $\vartheta_{i}-\vartheta_{j}=\left(\tau_{i}-\tau_{j}\right) \Delta$ on $\mathcal{H}_{1}$. Another way of saying this is that $\operatorname{det}_{i, j}(\vartheta, 1)=\vartheta_{i}-\vartheta_{j}=\left(\tau_{i}-\tau_{j}\right) \Delta=\operatorname{det}_{i, j}(\tau, 1) \Delta$ on $\mathcal{H}_{1}$. Using $\tau_{0}=0$ we have $\vartheta_{i}=\vartheta_{0}+\tau_{i} \Delta$ with $\vartheta_{0}$ being the theta series of the Leech lattice. It follows that $\operatorname{det}_{i, j, k}(\vartheta, \tau, 1)$ is identically zero on $\mathcal{H}_{1}$ because the first row is a linear combination of the second and third rows in the determinant; hence it is a cusp form 


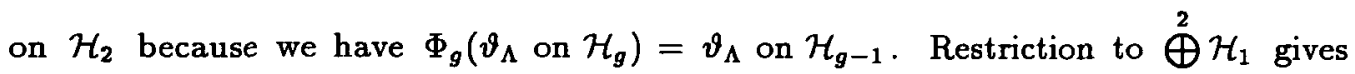
$\vartheta_{i}=\left.\left.\vartheta_{i}\right|_{\mathcal{H}_{1}} \otimes \vartheta_{i}\right|_{\mathcal{H}_{1}}=\vartheta_{0} \otimes \vartheta_{0}+\tau_{i}\left(\vartheta_{0} \otimes \Delta+\Delta \otimes \vartheta_{0}\right)+\tau_{i}^{2} \Delta \otimes \Delta$ so that by subtracting multiples of the second and third rows from the first row in the determinant, we have $\left.\operatorname{det}_{i, j, k}(\vartheta, \tau, 1)\right|_{\bigoplus^{2} \mathcal{H}_{1}}=\operatorname{det}_{i, j, k}\left(\tau^{2} \Delta \otimes \Delta, \tau, 1\right)=\operatorname{det}_{i, j, k}\left(\tau^{2}, \tau, 1\right) \Delta \otimes \Delta$ as claimed

Without loss of generality and for convenience, we can number the Niemeier lattices so that the first five Coxeter numbers are distinct $\left[2\right.$, p.407] and $\tau_{0}=0$ still. Then $\operatorname{det}_{0,1,2}\left(\tau^{2}, \tau, 1\right) \neq 0$, so that we may let $\phi=\operatorname{det}_{0,1,2}(\vartheta, \tau, 1) / \operatorname{det}_{0,1,2}\left(\tau^{2}, \tau, 1\right)$. Since $\operatorname{det}_{0,1,2}(\vartheta, \tau, 1)=\operatorname{det}_{0,1,2}\left(\tau^{2}, \tau, 1\right) \Delta \otimes \Delta$ on $\stackrel{2}{\oplus} \mathcal{H}_{1}$, we have $\phi=\Delta \otimes \Delta$ on $\stackrel{2}{\oplus} \mathcal{H}_{1}$. This implies $\phi$ is not identically zero on $\stackrel{2}{\oplus} \mathcal{H}_{1}$, and hence $\phi \neq 0$ in $S_{1}^{12}$. Since $\operatorname{dim} S_{2}^{12}=1$, $\phi$ must be a generator. So on $\mathcal{H}_{2}$, we must have

$$
\operatorname{det}_{i, j, k}(\vartheta, \tau, 1)=s_{i j k} \phi
$$

for some constant $s_{i j k}$. We also know that $\operatorname{det}_{i, j, k}(\vartheta, \tau, 1)=\operatorname{det}_{i, j, k}\left(\tau^{2}, \tau, 1\right) \Delta \otimes \Delta$ on $\stackrel{2}{\oplus} \mathcal{H}_{1}$. From (2.2), we also have that $\operatorname{det}_{i, j, k}(\vartheta, \tau, 1)=s_{i j k} \Delta \otimes \Delta$ on $\stackrel{2}{\oplus} \mathcal{H}_{1}$. So we conclude that $s_{i j k}=\operatorname{det}_{i, j, k}\left(\tau^{2}, \tau, 1\right)$. So on $\mathcal{H}_{2}$, we have $\operatorname{det}_{i, j, k}(\vartheta, \tau, 1)=$ $\operatorname{det}_{i, j, k}\left(\tau^{2}, \tau, 1\right) \phi$. Expanding the determinant by cofactors using the second row $\left(\tau^{2}\right)$, we have on $\mathcal{H}_{2}$,

$$
\begin{aligned}
& \underset{i_{1}, i_{2}, i_{3}, i_{4}}{\operatorname{det}}\left(\vartheta, \tau^{2}, \tau, 1\right)=\sum_{k=1}^{4}(-1)^{k+1} \tau_{i_{i_{k}}}^{2} \operatorname{det}_{\substack{i_{1}, \ldots, i_{4} \\
\text { no } i_{k}}}^{\operatorname{det}}(\vartheta, \tau, 1) \\
& =\sum_{k=1}^{4}(-1)^{k+1} \tau_{i_{k}}^{2} \operatorname{det}_{\substack{i_{1}, \ldots, i_{4} \\
\text { no } i_{k}}}\left(\tau^{2}, \tau, 1\right) \phi \\
& =\operatorname{det}_{i_{1}, i_{2}, i_{3}, i_{4}}\left(\tau^{2}, \tau^{2}, \tau, 1\right) \phi \\
& =0 \text {. }
\end{aligned}
$$

Therefore, $\operatorname{det}_{i, j, k, l}\left(\vartheta, \tau^{2}, \tau, 1\right)$ is a cusp form on $\mathcal{H}_{3}$. Restriction to $\stackrel{3}{\oplus} \mathcal{H}_{1}$ gives $\vartheta_{i}=$ $\vartheta_{i} \otimes \vartheta_{i} \otimes \vartheta_{i}=\vartheta_{0} \otimes \vartheta_{0} \otimes \vartheta_{0}+\cdots+\tau_{i}^{3} \Delta \otimes \Delta \otimes \Delta$ so that $\left.\operatorname{det}_{i, j, k, l}\left(\vartheta, \tau^{2}, \tau, 1\right)\right|_{\bigoplus^{3} \mathcal{H}_{1}}=$ $\operatorname{det}_{i, j, k, l}\left(\Delta \otimes \Delta \otimes \Delta \tau^{3}, \tau^{2}, \tau, 1\right)=\operatorname{det}_{i, j, k, l}\left(\tau^{3}, \tau^{2}, \tau, 1\right) \Delta \otimes \Delta \otimes \Delta$.

Since $\operatorname{dim} S_{3}^{12}=1[14, \mathrm{p} .832]$ and since we have four distinct Coxeter numbers $\tau_{0}, \tau_{1}, \tau_{2}, \tau_{3}$, we may employ the same linear algebra techniques as above to deduce that $\operatorname{det}_{i, j, k, l, m}\left(\vartheta, \tau^{3}, \tau^{2}, \tau, 1\right)$ is identically zero on $\mathcal{H}_{3}$ and is a cusp form on $\mathcal{H}_{4}$. Restriction to $\stackrel{4}{\oplus} \mathcal{H}_{1}$ gives $\left.\operatorname{det}_{i, j, k, l, m}\left(\vartheta, \tau^{3}, \tau^{2}, \tau, 1\right)\right|_{\bigoplus^{4} \mathcal{H}_{1}}=\operatorname{det}_{i, j, k, l, m}\left(\tau^{4}, \tau^{3}, \tau^{2}, \tau, 1\right) \Delta \otimes$ $\Delta \otimes \Delta \otimes \Delta$ in the same manner as above. 
The simple pattern of this Lemma does not continue because $\operatorname{dim} S_{4}^{12}=2$, as we shall deduce from a Theorem of Erokhin $[3,4]$.

TheOREM 2.3. (Erokhin) We have $\operatorname{dim}_{\mathbb{C}} \operatorname{Span}\left\{\vartheta_{i}\right.$ on $\left.\mathcal{H}_{4}: i \in\{0,1, \ldots, 23\}\right\}=$ 6.

Proof: In the notation of [4] the assertion of the Theorem is $\operatorname{dim} \operatorname{Im} \phi_{4}=6$, where $\phi_{g}: \mathbb{C}^{24} \rightarrow M_{g}^{12}$ is defined by $c \mapsto \sum_{i} c_{i} \vartheta_{i}$. Let $V_{g}=\left\{v \in \mathbb{C}^{24}: \forall c \in \operatorname{ker} \phi_{g},{ }^{t} v c=0\right\}$, then we have $\operatorname{dim} V_{g}=\operatorname{dim} \operatorname{Im} \phi_{g}$. Corollary 1 of [4, p.1017] and Theorem 2 of [4, p.1018] assert that $V_{4}$ has a basis of six elements. (These are $\left\{1, v_{A_{1}}, v_{A_{1}}^{2}, v_{A_{1}}^{3}, v_{A_{1}}^{4}, v_{D_{4}}\right\}$ in the notation of [4].)

Corollary 2.4. We have $\operatorname{dim} M_{4}^{12}=6, \operatorname{dim} S_{4}^{12}=2$.

Proof: From Theorem 1.2, and $12>2 \cdot 4$, we see that the theta series of the Niemeier lattices span $M_{4}^{12}$ and so $\operatorname{dim} M_{4}^{12}=6$ using the previous Theorem of Erokhin. From the surjectivity of $\Phi_{g}: M_{g}^{k} \rightarrow M_{g-1}^{k}$ for even $k>2 g[12$, p.68] the following sequence of complex vector spaces is exact: $0 \rightarrow S_{4}^{12} \rightarrow M_{4}^{12} \rightarrow M_{3}^{12} \rightarrow 0$. Since $\operatorname{dim} M_{3}^{12}=4[14, \mathrm{p} .835]$ we have $\operatorname{dim} S_{4}^{12}=2$.

Proposition 2.5. For any five indices $i, j, k, l, m \in\{0,1, \ldots, 23\}$ such that the Coxeter numbers $\tau_{i}, \tau_{j}, \tau_{k}, \tau_{l}, \tau_{m}$ are distinct, we have that $S_{4}^{12}$ is spanned by $f_{4} j_{8}$ and $\operatorname{det}_{i, j, k, l, m}\left(\vartheta, \tau^{3}, \tau^{2}, \tau, 1\right)$.

ProOF: Since $\operatorname{dim} S_{4}^{12}=2$ by Corollary 2.4 it suffices to show that the cusp forms $f_{4} j_{8}$ and $\operatorname{det}_{i, j, k, l, m}\left(\vartheta, \tau^{3}, \tau^{2}, \tau, 1\right)$ are linearly independent. By Lemma 2.1 the cusp form $\operatorname{det}_{i, j, k, l, m}\left(\vartheta, \tau^{3}, \tau^{2}, \tau, 1\right)$ does not vanish on the diagonal locus whereas $f_{4} j_{8}$ does because $j_{8}$ vanishes on the Jacobian locus and hence on the diagonal locus.

Theorem 2.6. We have $\operatorname{dim} S_{4}^{8}=1$ and $j_{8} \operatorname{spans} S_{4}^{8}$.

Proof: Since there exist five Niemeier lattices with distinct Coxeter numbers [2, p.407], let $\psi_{12}=\operatorname{det}_{i, j, k, l, m}\left(\vartheta, \tau^{3}, \tau^{2}, \tau, 1\right)$ be such that $f_{4} j_{8}$ and $\psi_{12}$ span $S_{4}^{12}$ as in Proposition 2.5. Take any $f \in S_{4}^{8}$. By Theorem $1.3 f$ must vanish on the hyperelliptic locus and hence on the diagonal locus. We also have $f_{4} f \in S_{4}^{12}$ and so $f_{4} f=a f_{4} j_{8}+b \psi_{12}$ for some $a, b \in \mathbb{C}$. Upon restriction to $\stackrel{4}{\oplus} \mathcal{H}_{1}$ we obtain $0=b \operatorname{det}\left(\tau^{4}, \tau^{3}, \tau^{2}, \tau, 1\right) \Delta \otimes \Delta \otimes \Delta \otimes \Delta$ by Lemma 2.1. Therefore $b=0$ and $f_{4} f=$ $a f_{4} j_{8}$. We conclude that $f=a j_{8}$ because the ring of Siegel modular forms has no zero divisors.

Corollary 2.7. We have $\operatorname{dim} M_{4}^{8}=2$.

Proof: Since $M_{3}^{8}$ is spanned by $f_{4}^{2}[9, \mathrm{p} .854]$, the map $\Phi_{4}: M_{4}^{8} \rightarrow M_{3}^{8}$ is onto and the sequence $0 \rightarrow S_{4}^{8} \rightarrow M_{4}^{8} \rightarrow M_{3}^{8} \rightarrow 0$ is exact. Hence we have $\operatorname{dim} M_{4}^{8}=2$. 
REMARK. We see that the theta series also span $M_{4}^{8}$ in this particular case where $k=2 g$.

ThEOREM 2.8. We have $S_{4}^{6}=0$.

Proof: Let the notation be as in the proof of Theorem 2.6. Any $f \in S_{4}^{6}$ vanishes on the hyperelliptic locus by Theorem 1.3 and hence on the diagonal locus. Since $f^{2} \in S_{4}^{12}$ we have $f^{2}=a f_{4} j_{8}+b \psi_{12}$, for some constants $a$ and $b$. Considering the restriction to $\oplus \mathcal{H}_{1}$, since $f$ and $j_{8}$ both vanish there and $\psi_{12}$ does not, we must have $b=0$. Thus we have $f^{2}=a f_{4} j_{8}$. There are two ways to show that $a=0$ and $f=0$, completing the proof. First, the ring of Siegel modular forms is a unique factorisation ring for $g \geqslant 3[7,8]$ and $j_{8}$ is irreducible by Theorem 1.4. Second, since $f^{2}=a f_{4} j_{8}$ vanishes on the Jacobian locus, so must $f$ itself. But since $j_{8}$ generates the ideal of forms vanishing on the Jacobian locus by Theorem 1.4, we must have that $f=f_{-2} j_{8}$ for some modular form $f_{-2}$ of weight $6-8=-2$, which is necessarily 0 .

REMARK. Since $\operatorname{dim} M_{3}^{6}=1[9, \mathrm{p} .852]$ this Theorem shows that either $\operatorname{dim} M_{4}^{6}=0$ or $\operatorname{dim} M_{4}^{6}=1$.

\section{REFERENCES}

[1] S. Böcherer, 'Über die Fourier-Jacobi-Entwicklung Siegelscher Eisensteinreihen', Math. Z. 183 (1983), 21-46.

[2] J.H. Conway, and N.J.A. Sloane, Sphere packings, lattices and groups, Grundlehren Math. Wiss. 290 (Springer-Verlag, Berlin, Heidelberg, New York, 1993).

[3] V. A. Erokhin, 'Theta series of even unimodular 24-dimensional lattices', LOMI 86 (1979), 82-93.

[4] V. A. Erokhin, 'Theta series of even unimodular lattices', LOMI 199 (1981), 59-70.

[5] J. Fay, Theta Functions on Riemann Surfaces, Springer Lecture Notes 352 (Springer-Verlag, Berlin, 1973).

[6] E. Freitag, Siegelsche Modulfunktionen, Grundlehren der mathematische Wissenschaften 254 (Springer Verlag, Berlin, 1983).

[7] E. Freitag, 'Stabile Modulformen', Math. Ann. 230 (1977), 197-211.

[8] E. Freitag, 'Die Irreduzibilität der Schottkyrelation (Bemerkung zu einen Satz von J. Igusa)', Arch. Math. 40 (1983), 255-259.

[9] J. I. Igusa, 'Modular forms and projective invariants', Amer. J. Math. 89 (1967), 817-855.

[10] J. I. Igusa, 'Schottky's invariant and quadratic forms', in Christoffel Symposium(Birkhäuser Verlag, 1981).

[11] J. I. Igusa, 'On the irreducibility of Schottky's divisor', Tokyo Imperial University Faculty of Science Journal Section IA 28 (1981).

[12] H. Klingen, Introductory lectures on Siegel modular forms, Cambridge Studies in Advanced Mathematics 20 (Cambridge University Press, Cambridge, 1990). 
[13] R. Tsuhima, 'On dimension Formula for Siegel Modular Forms', Automorphic Forms and Geometry of Arithmetic Varieties, Advanced Studies in Pure Mathematics 15 (Cambridge University Press, Cambridge, 1989), pp. 41-64.

[14] S. Tsuyumine, 'On Siegel modular forms of degree three', Amer. J. Math. 108 (1986), 755-862, 1001-1003.

Department of Mathematics

Fordham University

Bronx NY 10458

United States of America

e-mail: poor@murray.fordham.edu
Math/Computer Science Department

Lake Forest College

555 N. Sheridan Rd

Lake Forest IL 60045

United States of America

e-mail: yuen@math.lfc.edu 